\title{
An evidence-based practice-oriented review focusing on canagliflozin in the management of type 2 diabetes
}

This article was published in the following Dove Press journal:

Vascular Health and Risk Management

17 February 2017

Number of times this article has been viewed

\author{
Joseph A Messana' \\ Stanley S Schwartz ${ }^{2,3}$ \\ Raymond R Townsend' \\ 'Nephrology Division, Perelman \\ School of Medicine, University of \\ Pennsylvania, ${ }^{2}$ Main Line Health, \\ ${ }^{3}$ Department of Medicine, University \\ of Pennsylvania, Philadelphia, PA, USA
}

Correspondence: Raymond R Townsend Renal, Electrolyte and Hypertension Division, Perelman School of Medicine, University of Pennsylvania, 122 Founders Building, 3400 Spruce Street, Philadelphia, PA 19104, USA

Tel +I 2156140423

Fax +I 2156623459

Email townsend@upenn.edu
Abstract: Caring for patients with type 2 diabetes mellitus (T2DM) has entered an era with many recent additions to the regimens used to clinically control their hyperglycemia. The most recent class of agents approved by the Food and Drug Administration (FDA) for T2DM is the sodium-glucose-linked transporter type 2 (SGLT2) inhibitors, which work principally in the proximal tubule of the kidney to block filtered glucose reabsorption. In the few years attending this new class arrival in the market, there has been a great deal of interest generated by the novel mechanism of action of SGLT2 inhibitors and by recent large outcome trials suggesting benefit on important clinical outcomes such as death, cardiovascular disease and kidney disease progression. In this review, we focus on canagliflozin, the first-in-class marketed SGLT2 inhibitor in the USA. In some cases, we included data from other SGLT2 inhibitors, such as outcomes in clinical trials, important insights on clinical features and benefits, and adverse effects. These agents represent a fundamentally different way of controlling blood glucose and for the first time in T2DM care to offer the opportunity to reduce glucose, blood pressure, and weight with effects sustained for at least 2 years. Important side effects include genital mycotic infections and the potential for orthostatic hypotension and rare instances of normoglycemic ketoacidosis. Active ongoing clinical trials promise to deepen our experience with the potential benefits, as well as the clinical risks attending the use of this new group of antidiabetic agents.

Keywords: SGLT2, canagliflozin, review, outcomes, type 2 diabetes mellitus

\section{Background \\ Scope of problems in managing the patients with type 2 diabetes mellitus (T2DM)}

The obesity epidemic has had multiple effects on public health, including an increase in blood pressure (BP) and in the prevalence of T2DM..$^{1,2}$ Approximately one in seven adults in the USA have diabetes, and roughly two out of five have prediabetes based on the 2011 National Health and Nutrition Examination Survey data. ${ }^{3}$ Both diabetes and prediabetes substantially increase the risk of cardiovascular $(\mathrm{CV})$ and renal disease. ${ }^{4}$ Other risk factors for T2DM include age, physical inactivity, ethnicity, and medications such as glucocorticoids. ${ }^{5}$ Diabetes frequently conspires with hypertension and dyslipidemia predisposing patients to several additional CV risks. Moreover, the prevalence of T2DM among adolescents is increasing, ${ }^{6}$ and this is expected to increase by an additional $50 \%$ by $2050 . .^{7}$ Tragically, earlier onset and a longer duration of diabetes enhance the likelihood of microvascular and macrovascular disease manifestations, including coronary artery disease, stroke, peripheral artery disease, and kidney failure, 
which have dramatic effects on life quality, ability to work, and the costs of health care. ${ }^{7}$ Often, the elevation in glucose in T2DM smolders for a long period undetected, and patients can present with a diabetes-related complication at the time of the diagnosis of diabetes. ${ }^{8}$ Such findings support the clinical concept that tissue damage associated with hyperglycemia develops before the clinical symptoms and signs are manifest. ${ }^{9}$

\section{Evolution of therapies for diabetes}

The management of hyperglycemia in diabetic patients has fostered the development of an impressive array of lifestyle and pharmaceutical approaches. Among the lifestyle factors espoused by the American Diabetes Association, weight loss and exercise feature prominently. ${ }^{10}$ Among the pharmaceutical approaches other than insulin, metformin has been consistently recommended as a useful agent in T2DM in part because it does not promote weight gain or hypoglycemia, unlike sulfonylurea agents and insulin, which typically do. ${ }^{11}$ In recent years, a number of new classes of antihyperglycemic agents have been introduced in the management of T2DM as shown in Figure 1. One new class, which typically is associated with weight reduction, is the selective inhibitors of the sodium-glucose-linked transporter type 2 (SGLT2) located principally in the proximal tubule of the kidney. The first agent in this class approved by the Food and Drug Administration (FDA) for T2DM in the USA in 2013 was canagliflozin.

\section{Phlorizin: the earliest known SGLT2 inhibitor}

Phlorizin was first isolated from the bark of the apple tree by French chemists in $1835 .{ }^{12}$ Since the bark of other trees had proven useful as antipyretics and antimalarials, phlorizin was initially hoped to be such. Investigations revealed that it could lower elevated glucose levels when administered orally in a diabetic mouse model. The mechanisms became apparent in that phlorizin administration produced a mellituria (sweet urine) as first noted in 1885 . Hampered by nonselective SGLT1 and SGLT2 blockade, low oral availability, and rapid metabolism by $\beta$-glucosidase, interest in developing it as a human antihyperglycemic agent languished until the late 1980s when interest in sodium-glucose-linked transporters (SGLTs) began to intensify and studies revealed (again) that phlorizin could reduce glucose levels and improve insulin sensitivity in diabetic rats. ${ }^{12}$ Manipulation of the basic phlorizin structure with the addition of aryl rings increased the selectivity for SGLT2, ${ }^{13}$ and further refinements included adding an arylglucoside to reduce beta-glucosidase cleavage. ${ }^{14}$ The chemical structure of phlorizin is shown in Figure 2,

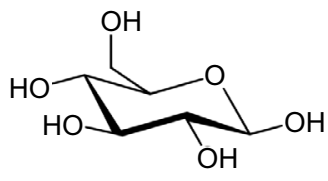

Glucose

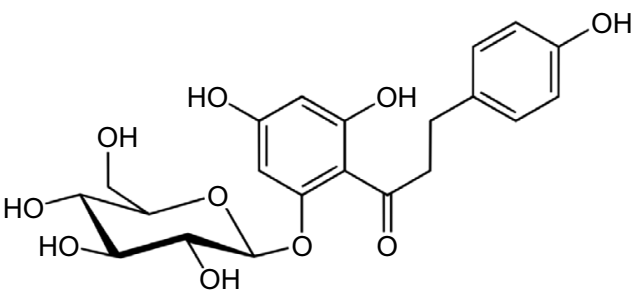

Phlorizin

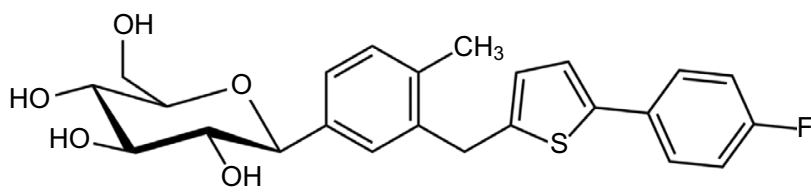

Canagliflozin

Figure $\mathbf{2}$ The chemical structures of phlorizin, glucose, and canagliflozin.

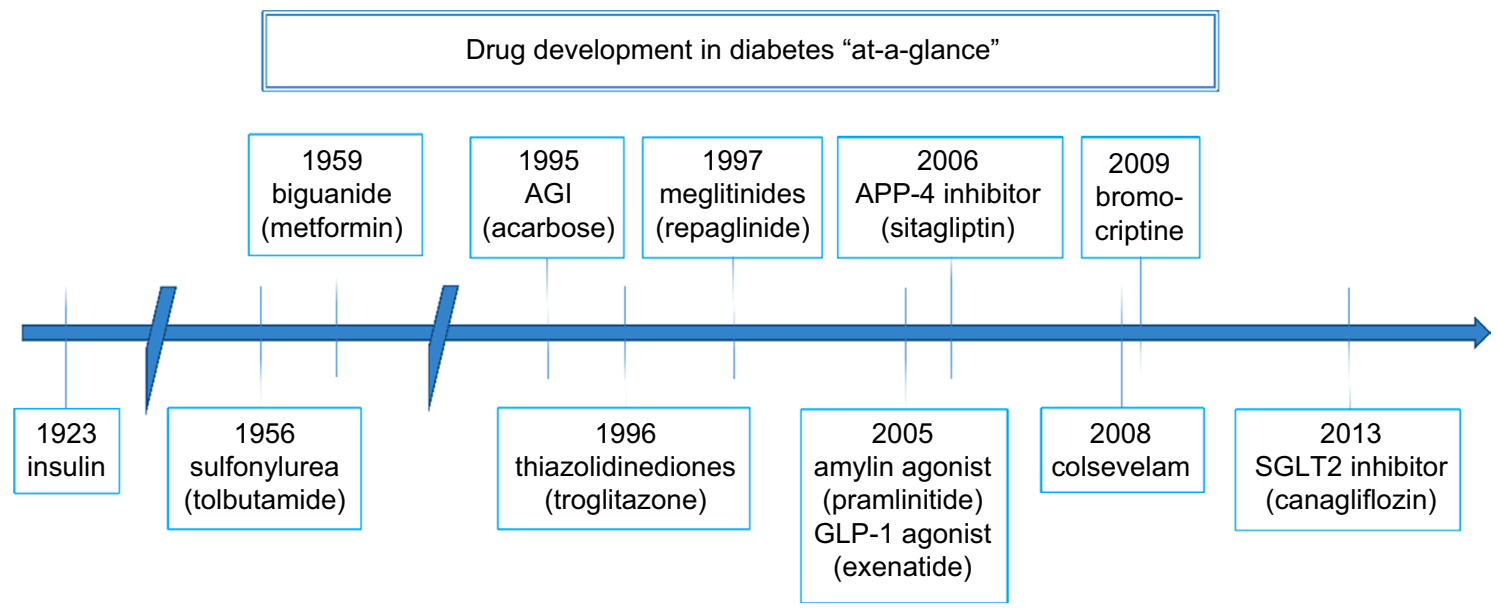

Figure I Timeline for milestones in the development of drugs to manage diabetes.

Abbreviations: AGI, alpha-glucosidase inhibitor; GLP, glucagon-like polypeptide; SGLT2, sodium-glucose-linked transporter type 2. 
with a glucose molecule placed beside it, along with the chemical structure of canagliflozin, so that the reader can see the similarities and how the molecular mimicry of phlorizin lends itself to occupying the SGLT2 inhibitor.

\section{Mechanisms of action of canagliflozin Renal glucosuria}

Normal human urine is essentially devoid of glucose. Consequently, measurable urinary glucose excretion (glucosuria) is usually abnormal; however, glucosuria occurs in a variety of contexts. When glucosuria is accompanied by excessive urinary excretion of amino acids, phosphate, bicarbonate, and other solutes, the diagnosis of Fanconi syndrome is likely present. If glucosuria is isolated, without hyperglycemia, this corroborates familial renal glucosuria (FRG) as the diagnosis. FRG is linked to mutation in the solute carrier family 5 (sodium/glucose transporter) member 2 (SLC5A2) gene, which implicates functional disruption or loss of function of the SGLT2. ${ }^{15}$ Daily glucosuria can range from $<1 \mathrm{~g} /$ day to as much as $150 \mathrm{~g} / 1.73 \mathrm{~m}^{2} /$ day. Given the multitude of potential mutations, the varied degree of penetrance, and the overall lack of phenotype, it is difficult to determine finite genotype-phenotype links. Generally, FRG is considered to be a benign condition as it is not associated with clinical consequences. In one study, after a follow-up time of 30 years, the only manifestations detected were polyuria, enuresis, and a later mild growth and pubertal maturation delay. ${ }^{16}$ Some other reported symptoms were episodic dehydration and ketosis, during times of starvation, and increased urinary tract infections. In a 104-week trial comparing canagliflozin with glimepiride, a significant reduction in estimated glomerular filtration rate (eGFR) was observed with glimepiride, whereas eGFR remained almost stable with canagliflozin $100 \mathrm{mg}$ and $300 \mathrm{mg} .{ }^{17}$ The overall benign nature of FRG suggests that the application of therapeutics that mimic the same molecular changes, namely SGLT2 inhibitors, would have a similar benign course.

\section{Physiology of kidney glucose transport}

Research into how the kidney recovers filtered glucose has identified two principal SGLTs in the proximal tubule, where virtually all of the glucose filtered by the glomerulus is recovered and most of that is through the SGLT2. ${ }^{18,19}$ Phlorizin, hampered by poor oral absorption and rapid metabolism, nonetheless demonstrated the proof of principle that blood sugar could be reduced through glucosuria, setting the stage for the development of the class of SGLT2 inhibitors, of which canagliflozin was the first FDA-approved agent in class in the US market in 2013. Others have followed, including dapagliflozin (FDA approved in January 2014) and empagliffozin (FDA approved in July 2014), with others in development.

Studies on the blocking effects of phlorizin on proximal kidney tubule reabsorptive function were specific for glucose. Phlorizin, and the subsequent SGLT2 inhibitors such as canagliflozin, did not affect amino acid excretion and appeared to model a disorder known as renal glycosuria, an autosomal dominant heritable condition. ${ }^{15}$

\section{Clinical profile of canagliflozin Clinical effects of canagliflozin Effects on glucose}

Distal tubules in the kidney are not equipped to handle glucose that escaped reabsorption proximally. Proximal SGLT2 blockade lowers blood glucose levels in a manner proportionate to the level of glucose filtered. In conjunction with the reduction in blood glucose, there is a dose-related decline in $\mathrm{HbA} 1 \mathrm{C}$ concentrations of $\sim 0.5 \%$ with the $100 \mathrm{mg}$ once-daily dose and $1.0 \%$ with the $300 \mathrm{mg}$ once-daily dose. Although estimates vary, it is likely that $300-500 \mathrm{kcal}$ of energy in the form of glucose are lost each day in a diabetic taking an SGLT2 inhibitor.

Table 1 lists a number of efficacy trials of canagliflozin used alone or in combination with other agents. At daily doses of $100 \mathrm{mg}$ and $300 \mathrm{mg}$ canagliflozin, therapy is typically associated with a reduction in glycated HbA1C compared with placebo on the order of $0.9-1.2 \%{ }^{20,21}$ When used in combination with metformin, the reductions in $\mathrm{HbA} 1 \mathrm{C}$ are typically $\sim 0.6 \%$. When used with metformin and a sulfonylurea, reductions of $\sim 0.7 \%$ are noted. The combination of canagliflozin to metformin plus thiazolidinedione is attended by $\sim 0.9 \% \mathrm{HbA} 1 \mathrm{C}$ reduction. ${ }^{22-25}$ Compared with glimepiride, reductions in $\mathrm{HbA} 1 \mathrm{C}$ with canagliflozin add-on therapy (decrease of $0.8-0.9 \%$ ) in patients already treated with metformin were similar to glimepiride (decrease of $0.8 \%)$ at 52 weeks. ${ }^{26}$ These changes in $\mathrm{HbA} 1 \mathrm{C}$ remained persistent for 2 years. ${ }^{27}$ In patients treated with metformin plus a sulfonylurea, reductions in HbA1C with $300 \mathrm{mg}$ canagliflozin daily $(1.0 \%)$ were similar to sitagliptin $(0.7 \%)$ at 52 weeks. ${ }^{28}$ As monotherapy, or in combination regimens, canagliflozin is an effective antihyperglycemic agent.

There are limited data on SGLT1 selectivity differences between current SGLT2 inhibitors on the market. It is postulated that greater selectivity of canagliflozin for SGLT1 may reduce postprandial sugars more effectively than others 
Table I Representative clinical trials of glucose control and body weight changes with canagliflozin

\begin{tabular}{|c|c|c|c|c|c|c|}
\hline Study & Therapy & Treatment arms (n) & $\begin{array}{l}\text { Baseline AIC\% } \\
\text { at baseline } \\
(\text { mean } \pm \text { SD) }\end{array}$ & $\begin{array}{l}\text { Change in } \\
\text { AlC\% }\end{array}$ & $\begin{array}{l}\text { Baseline } \\
\text { body weight } \\
\text { (kg; mean } \pm \text { SD) }\end{array}$ & $\begin{array}{l}\text { Change in body } \\
\text { weight }(\mathrm{kg})\end{array}$ \\
\hline \multirow[t]{3}{*}{ Stenlof et $\mathrm{a}^{20}$} & Mono-Rx, 26 weeks & CANA $100 \mathrm{mg}(195)$ & $8.1 \pm 1.0$ & $-0.77(P<0.00 \mathrm{I})$ & $85.8 \pm 21.4$ & $-2.5(P<0.001)$ \\
\hline & & CANA 300 mg (197) & $8.0 \pm 1.0$ & $-1.03(P<0.001)$ & $86.9 \pm 20.5$ & $-3.4(P<0.001)$ \\
\hline & & PBO (192) & $8.0 \pm 1.0$ & 0.14 & $87.6 \pm 19.5$ & -0.5 \\
\hline \multirow[t]{3}{*}{ Wilding et $\mathrm{a}^{25}$} & $\begin{array}{l}\text { Combo-Rx, MET + SU, } \\
26 \text { weeks }\end{array}$ & CANA 100 mg (I57) & $8.1 \pm 0.9$ & $-0.85(P<0.00 I)$ & $93.8 \pm 22.6$ & $-1.9(P<0.00 \mathrm{I})$ \\
\hline & & CANA 300 mg (156) & $8.1 \pm 0.9$ & $-1.06(P<0.001)$ & $93.5 \pm 22.0$ & $-2.5(P<0.00 \mathrm{I})$ \\
\hline & & PBO (I56) & $8.1 \pm 0.9$ & -0.13 & $91.2 \pm 22.6$ & -0.8 \\
\hline Lavalle- & Combo-Rx + MET, & CANA 100 mg (368) & $7.9 \pm 0.9$ & $-0.79(P<0.001)$ & $88.8 \pm 22.2$ & $-3.3(P<0.001)$ \\
\hline \multirow[t]{5}{*}{ Gonzalez et $\mathrm{a}^{23}$} & 26 weeks & & & & & \\
\hline & & CANA 300 mg (367) & $7.9 \pm 0.9$ & $-0.94(P<0.00 I)$ & $85.4 \pm 20.9$ & $-3.6(P<0.001)$ \\
\hline & & SITA 100 mg (366) & $7.9 \pm 0.9$ & -0.82 & $87.7 \pm 21.6$ & -1.1 \\
\hline & & & & NA & & NT \\
\hline & & PBO (I83) & $8.0 \pm 0.9$ & -0.17 & $86.6 \pm 22.4$ & -1.1 \\
\hline \multirow[t]{5}{*}{ Cefalu et $\mathrm{a}^{26}$} & $\begin{array}{l}\text { Combo-Rx + MET, } \\
52 \text { weeks }\end{array}$ & CANA 100 mg (483) & $7.8 \pm 0.8$ & -0.82 & $86.9 \pm 20.1$ & $-3.7(P<0.000 \mathrm{I})$ \\
\hline & & & & NA & & \\
\hline & & CANA 300 mg (485) & $7.8 \pm 0.8$ & -0.93 & $86.6 \pm 19.5$ & $-4.0(P<0.001)$ \\
\hline & & & & NA & & \\
\hline & & GLIM 6-8 mg (484) & $7.8 \pm 0.8$ & $-0.8 \mathrm{I}$ & $86.5 \pm 19.8$ & 0.7 \\
\hline \multirow[t]{4}{*}{$\begin{array}{l}\text { Schernthaner } \\
\text { et } \mathrm{a}^{28}\end{array}$} & $\begin{array}{l}\text { Combo-Rx MET + SU, } \\
52 \text { weeks }\end{array}$ & CANA 300 mg (378) & $8.1 \pm 0.9$ & -1.03 & $87.4 \pm 23.2$ & $-2.3(P<0.00 \mathrm{I})$ \\
\hline & & & & NA & & \\
\hline & & SITA 100 mg (378) & $8.1 \pm 0.9$ & -0.66 & $89.1 \pm 23.2$ & 0.1 \\
\hline & & & & NA & & \\
\hline \multirow[t]{2}{*}{ Inagaki et $\mathrm{al}^{21}$} & Mono-Rx, 24 weeks & CANA 100 mg $(90)$ & $7.98 \pm 0.73$ & $-0.74(P<0.00 I)$ & $69.1 \pm 14.5$ & $-3.8(P<0.00 \mathrm{I})$ \\
\hline & & PBO (93) & $8.04 \pm 0.7$ & 0.29 & $68.6 \pm 15.2$ & -0.8 \\
\hline \multirow[t]{3}{*}{ Forst et $\mathrm{a}^{22}$} & $\begin{array}{l}\text { Combo-Rx, MET + PIO, } \\
26 \text { weeks }\end{array}$ & CANA $100 \mathrm{mg}(1 \mathrm{I})$ & $8.0 \pm 0.9$ & $-0.89(P<0.00 \mathrm{I})$ & $94.2 \pm 22.2$ & $-2.6(P<0.00 \mathrm{I})$ \\
\hline & & CANA $300 \mathrm{mg}(114)$ & $7.9 \pm 0.9$ & $-1.03(P<0.00 I)$ & $94.4 \pm 25.9$ & $-3.7(P<0.001)$ \\
\hline & & PBO/SITA 100 mg (II5) & $8.0 \pm 1.0$ & -0.26 & $93.8 \pm 22.4$ & -0.2 \\
\hline
\end{tabular}

Note: Sodium-glucose cotransporter 2 inhibitors: an evidence-based practice approach to their use in the natural history of type 2 diabetes, Schwartz SS, Ahmed I, Curr Med Res Opin, 2016;32(5):907-919, reprinted by permission of the publisher (Taylor \& Francis Ltd, http://www.tandfonline.com).$^{70}$

Abbreviations: CANA, canagliflozin; GLIM, glimepiride; PBO, placebo; SITA, sitagliptin; MET, metformin; SU, sulfonylurea; NA, not applicable; NT, not tested; PIO, pioglitazone; AIC\%, Hemoglobin AIC concentration.

in normal subjects. However, the importance of SGLT1 blockade in patients with T2DM has not been shown. We anticipate that, in the future, more comparative effectiveness investigations between agents will be undertaken given the recent publication of the framework for a network analysis of agents within the class. ${ }^{29}$

\section{Effects on weight}

The glucose-driven diuresis with canagliflozin represents a caloric drain on the body. In patients treated with canagliflozin for 6-12 months, weight reductions in the range of $\sim 3 \mathrm{~kg}$ occur with canagliflozin, which are maintained while treatment continues. ${ }^{30}$ The amount of weight loss is dose related. In a pooled analysis of 2250 patients treated with $100 \mathrm{mg}$ daily or $300 \mathrm{mg}$ daily of canagliflozin (or placebo) for 26 weeks, the $100 \mathrm{mg}$ dose was associated with $\sim 3 \%$ reduction in body weight and the $300 \mathrm{mg}$ dose with $\sim 4 \%$ reduction in body weight, while placebo had little effect. With canagliflozin treatment, approximately two-thirds of the weight loss is accounted for by a reduction in fat mass. ${ }^{31}$ Weight loss is likely part of the long-term reduction in BP noted with SGLT2 inhibitors. ${ }^{32}$ In a recent regression analysis, $\sim 42 \%$ of the placebo-subtracted reductions in systolic BP (SBP) with canagliflozin was dependent upon the reduction in weight. ${ }^{31}$

\section{Effects on BP}

As a class, SGLT2 inhibitors have shown a reasonably consistent effect on office and ambulatory BP. ${ }^{33}$ A pooled analysis of phase 3 studies comprising 2313 patients treated with canagliflozin again showed dose-related reductions in $\mathrm{BP}$, whether or not the patient was hypertensive and taking antihypertensive medications. ${ }^{34}$ In the normotensive patients, the reductions in BP were $\sim 4 / 2 \mathrm{mmHg}$ (systolic/diastolic) for the $100 \mathrm{mg}$ daily dose and $5 / 2 \mathrm{mmHg}$ for the $300 \mathrm{mg}$ 
daily dose more than placebo. In the hypertensive patients, the reductions with $100 \mathrm{mg}$ once daily were $6 /-1 \mathrm{mmHg}$ (diastolic was $\sim 1 \mathrm{mmHg}$ higher than placebo) and $7 / 2 \mathrm{mmHg}$ with $300 \mathrm{mg}$ once daily. A 6-week study of canagliflozin in 169 hypertensive diabetic patients comparing placebo with the $100 \mathrm{mg}$ and $300 \mathrm{mg}$ daily doses using 24-h ambulatory BP monitoring over a 6-week treatment period was recently reported. ${ }^{35}$ Measurements of 24-h ambulatory BP monitoring were undertaken prior to randomizing to the three arms, repeated on the day subjects took their first dose of blinded study medication, and performed a third time at the end of 6 weeks of treatment. Compared with placebo, 24-h BP values on $100 \mathrm{mg}$ daily were $3 / 2 \mathrm{mmHg}$ lower and with $300 \mathrm{mg}$ daily $5 / 3 \mathrm{mmHg}$ lower at the end of 6 weeks of therapy. Moreover, 24-h BP values were $2 \mathrm{mmHg}$ systolic lower on both doses of canagliflozin compared with placebo during the first day and night of blinded therapy when subjects received an SGLT2 inhibitor for the first time. The mechanisms of SGLT2 inhibition that lower BP include a volume reduction component, ${ }^{36}$ weight loss,${ }^{31}$ and possibly arterial stiffness reduction. ${ }^{30}$

\section{Effects on the sympathetic nervous system}

Our interpretation of the lack of heart rate increases despite the decline in BP and modest volume depletion in patients treated with SGLT2 inhibitors suggests that there may be an element of sympathetic inhibition in the mechanism of action of this class of drugs, but there is little beyond our speculation to support this, although we note that the reduction in $\mathrm{BP}$ values with SGLT2 inhibitors is generally not attended by an increase in heart rate. This supports an anti-sympathetic influence.

\section{Effects on the kidney function and albuminuria}

Kidney histological studies of diabetic animals treated with phlorizin revealed improved antioxidant enzyme activity and attenuated end effects of hyperglycemia. Diabetes is associated with increased tyrosine nitration in the renal cortex and medulla. Nitrotyrosine is presumed to be a stable end product of mitochondrial and cytosolic proteins from peroxynitrite. This modification represents either a marker of oxidative stress or the stable end product of mitochondrial and cytosolic protein alteration. ${ }^{37}$ In phlorizin-treated animals, the nitrotyrosine stain was decreased suggesting a protective effect. Phlorizin has also been shown to prevent proteinuria, hyperfiltration, and kidney hypertrophy, but not glomerular hypertrophy. ${ }^{38}$

SGLT2 inhibitors have been observed to promote reductions in intraglomerular pressure, and uric acid levels induced by SGLT2 inhibition in addition to decreased glomerular hyperfiltration and albuminuria. ${ }^{39}$ Tubuloglomerular feedback explains the effects of SGLT2 inhibition and the resulting mitigation of glomerular hyperfiltration. By increasing distal sodium delivery and thus sodium detection by the macular densa, adenosine is upregulated, resulting in afferent renal vasoconstriction and decreased glomerular filtration rate (GFR). ${ }^{40}$ A 6-month study of canagliflozin decreased the urinary albumin to creatinine ratio (ACR) in a study by Yale et $\mathrm{al}^{41}$ in a dose-dependent fashion, with the median urinary ACR decreasing by $29.9 \%, 20.9 \%$, and $7.5 \%$ in canagliflozin $100 \mathrm{mg}, 300 \mathrm{mg}$, and placebo groups, respectively.

Canagliflozin reduces urinary albumin excretion compared with other antihyperglycemics in diabetic patients already on metformin. ${ }^{42}$ The magnitude of urine ACR reduction appears to be time dependent and is $\sim 25 \%$ after 1 year, dropping to $\sim 10 \%$ at 2 years. Decreasing urinary albumin excretion is reassuring in terms of renal injury and suggests a protective effect.

\section{Effects on uric acid}

A recent meta-analysis of four studies observed that canagliflozin reduced serum uric acid concentrations by $13 \%$ (or a decrease in $0.7 \mathrm{mg} / \mathrm{dL}$ ) without a difference in the $100 \mathrm{mg}$ or $300 \mathrm{mg}$ daily doses. ${ }^{43}$ The mechanism for this is not clear at this time, but may be due to the solute carrier family 2 (facilitated glucose transporter) member 9 (SLCA2A9; also called "GLUT9"), a transporter that exchanges uric acid for glucose in the kidney tubule. ${ }^{44}$ Reductions in uric acid levels are associated with benefit in diabetic models, ${ }^{45}$ which suggests that this feature may be of clinical benefit.

\section{Adverse effects of SGLT2 inhibitors}

Although the primary focus of this review is the first-in-class SGLT2 inhibitor, canagliflozin, enough data have accumulated on this new class that the authors felt it important to cast a wide net in this section and cover the entire class.

\section{Hypoglycemia}

Although the risk of hypoglycemia with SGLT2 inhibitors is greater than placebo, ${ }^{46}$ it is in fact lower when compared with sulfonylureas. Dapagliflozin was found to have episodes of hypoglycemia at a rate of $11.8 \%$, which was marginally increased over placebo at $7.0 \% .{ }^{47}$ Protection from significant hypoglycemia occurs secondary to the insulin-independent mechanism of SGLT2 inhibitors, hepatic gluconeogenesis, and pharmacokinetics of the medicines. ${ }^{48}$ Diminished GFR is a limiting factor for the effectiveness of SGLT2 inhibitors 
and thus can dampen the degree of glucose lowering. The EMPA-REG OUTCOME study also revealed that the incidence of hypoglycemic events was comparable between placebo and empagliflozin at different doses and that empagliflozin in fact had less incidence of hypoglycemia requiring assistance. ${ }^{49}$ Overall, episodes of hypoglycemia occur at a similar rate as placebo and should not limit the use of SGLT2 inhibitors.

Interestingly, recent studies suggest an augmentation in insulin sensitivity and increased endogenous glucose production due to empagliflozin therapy. This may be due to decreased glucose toxicity but provides another compelling argument for the therapeutic potency of SGLT2 inhibitors. ${ }^{50}$

The risk of hypoglycemia is increased when SGLT2 inhibitors are combined with hypoglycemic agents (sulfonylureas or insulin) but can be proactively reduced by reducing or stopping other hypoglycemic therapy.

\section{Orthostatic BP changes}

Assuming the importance of the diuretic mechanism driving BP improvement under SGLT2 inhibition, there is concern for potentially threatening volume depletion. This fear restricts general utilization of SGLT2 inhibitors. The diuretic component of SGLT2 inhibitors is linked to the subsequent increase in sodium excretion. Although the risk of orthostasis exists, it is typically a transient symptom that may be exacerbated by the use of renin-angiotensin-aldosterone system inhibition. Orthostasis has been observed as a possible side effect of SGLT2 inhibitors, but it is infrequent. In a study of canagliflozin, $8 \%$ of test subjects were noted to have had transient orthostasis. ${ }^{51}$ This is in line with what is observed in FRG.

To investigate the diuretic properties of SGLT2 inhibitors, dapagliflozin was compared with placebo and hydrochlorothiazide in a randomized, double-blind trial. ${ }^{36}$ It revealed that the 24-h BP decrease for dapagliflozin was intermediate between the other two arms of the study with a decrease of $-0.9 \mathrm{mmHg}(95 \%$ confidence interval [CI]: $-4.2,+2.4)$, $-3.3 \mathrm{mmHg}(95 \% \mathrm{CI}:-6.8,+0.2)$, and $-6.6 \mathrm{mmHg}(95 \%$ CI: $-9.9,-3.2)$ for placebo, dapagliflozin, and hydrochlorothiazide, respectively. Curiously, office BP recordings were the lowest for dapagliflozin. The authors believed this to be secondary to BP recordings being taken during the daytime hours. Subgroup analyses were conducted to evaluate changes in body volume as well. Both plasma volume and red cell mass were estimated using radioisotope techniques with ${ }^{51} \mathrm{Cr}$ labeled erythrocytes and ${ }^{125}$ I-labeled human serum albumin. Changes from baseline in plasma volume for placebo, dapagliflozin, and hydrochlorothiazide groups were $+5.2 \%(-2.5$ to +8.7$),-7.3 \%(-12.4$ to -4.8$)$, and $+2.8 \%(-10.6$ to +25.7$)$, respectively. This corroborates that it is a unique quality of SGLT2 inhibitors to effectively decrease circulating plasma volume. However, the study also revealed a paradoxical slight increase in brain natriuretic peptide in the dapagliflozin arm. The authors attributed this potentially to increased erythropoietin, which has been linked to elevated brain natriuretic peptide levels. In addition, laboratories evaluated at 12 weeks also revealed an increase in plasma renin and aldosterone. This trend was also observed for canagliflozin, showing a modest increase in urine volume in week 1 out of a 12-week investigation. There was an attenuation of urine volume by week 12, but other indices such as creatinine and serum blood urea nitrogen remained elevated throughout. ${ }^{52}$

Proactively, if the patient's BP is on the low side when initiating SGLT2 inhibitor therapy, decreasing or stopping the diuretic, or another antihypertensive agent, can reduce the likelihood of symptomatic orthostasis.

\section{Acute kidney injury}

Given the BP effects during SGLT2 inhibition, and the feared diuretic component therein, there is a theoretical risk of significant hypotension leading to acute kidney injury. However, in the EMPA-REG study, the incidence of acute kidney injury was actually decreased in the empagliflozin arm when compared with the placebo group. ${ }^{49}$ Significant acute kidney injury seems to occur during episodes of euglycemic diabetic ketoacidosis (DKA), which are less common events. This suggests that the risk of acute kidney injury is minimal under SGLT2 inhibition.

\section{Hyperlipidemia and steatohepatitis}

SGLT2 inhibitors have been shown to affect lipid levels. Small increases in both high-density lipoprotein (HDL) and low-density lipoprotein (LDL) were observed, whereas triglycerides were mildly reduced under SGLT2 inhibition. LDL was increased from $1.8 \%$ to $4.4 \%$ with dapagliflozin versus $0.4 \%$ with placebo. Triglycerides were slightly reduced by $-2.4 \%$ to $-6.2 \%$ and by $2.1 \%$ with dapagliflozin and placebo, respectively. ${ }^{53}$ It is unclear if these small perturbations are clinically relevant. ${ }^{54}$ However, SGLT2 inhibition is simultaneously associated with overall weight reduction, decrease in adiposity, and attenuated degree of inflammation. As such, empagliflozin has been demonstrated to decrease the incidence of steatohepatitis. ${ }^{55}$ All SGLT2 inhibitors have been linked to waist circumference reduction. ${ }^{56}$ Thus, it is surprising that a small increase in LDL was detected; this observation may not be replicated on repeat investigations. 


\section{Infections}

The glucosuria resulting from SGLT2 inhibitor therapy has both positive and negative health benefits. Among the adverse effects noted with glucosuria is a susceptibility to urinary tract infections and genital mycotic infections. In a different double-blind, placebo-controlled, dose-ranging study, investigators revealed an increased risk of vulvovaginal adverse events (VVAEs) among those taking canagliflozin. ${ }^{57}$ Subjects were tested at baseline for Candida colonization, and of those who were initially negative, $31 \%$ of canagliflozin cohort and $14 \%$ of the placebo/sitagliptin subjects had conversion to positive urine tests by week 12 (odds ratio [OR], 2.8; 95\% CI, 1.0-7.3 for canagliflozin vs placebo/sitagliptin). This conversion placed subjects at increased risk for VVAEs, including candidiasis, cervicitis, furuncle, genital rash, vaginal discharge, vulvovaginal candidiasis, vulvovaginal mycotic infection, vulvovaginal pruritus, and vulvovaginitis. Two placebo/sitagliptin (3\%) and 16 canagliflozin subjects (10\%) experienced VVAE. Notably, these infections did not require discontinuation of the SGLT2 inhibitor, and they were simply treated with antifungal therapy. The EMPA-REG study also corroborates this phenomenon, with an increased percentage of genital infections in the empagliflozin group. ${ }^{49}$ Hasan et $\mathrm{al}^{14}$ found a dose-dependent increase in glucosuria; however, there did not appear to be a dose-dependent increase in urinary tract infections..$^{57}$

Thus, although there is an increase in urinary tract infections and genital mycotic infections, these seem to be easily treated, can be prospectively mitigated by advising fastidious bathroom habits, and do not outweigh the significant improvements in glucose control without hypoglycemia. In addition, one can further minimize infection risks by advising patients to drink more fluids - enough to keep urine dilute.

\section{Bone health and fracture risk}

In a randomized, double-blind study, 252 patients were assigned to either low-dose dapagliflozin, high-dose dapagliflozin, or placebo. It was found that 13 patients receiving dapagliflozin incurred a bone fracture, whereas no patients receiving placebo experienced a bone fracture. ${ }^{58}$ Seven of these 13 patients had diabetic nephropathy and orthostatic hypotension. Separately, it has been observed that there was an increased risk of fracture in a cohort taking canagliflozin after a mean duration of 68 weeks. ${ }^{59}$ Although the SGLT2 protein is poisoned, there is an increased sodium load in the renal tubule, which increases cotransport of sodium and phosphate through their respective protein. This leads to hyperphosphatemia and subsequently increased parathyroid hormone levels. ${ }^{60}$ The effects on 1,25-dihydroxyvitamin D is less clear as SGLT2 inhibitors increase both PTH and FGF23, which increase and decrease 1,25-dihydroxyvitamin D, respectively. These changes occurred while taking SGLT2 inhibitors at expected pharmacological dosing. Both dapagliflozin and canagliflozin have been shown to increase levels of bone formation biomarkers, corroborating increased bone turnover. Decreases in bone density may also attend the concurrent weight loss with SGLT2 inhibitors. ${ }^{61}$ In September 2015, the FDA strengthened its warning about canagliflozin and the increased risk of bone fractures.

\section{Ketoacidosis}

Several case reports have been generated describing cases of euglycemic DKA in the context of SGLT2 inhibitor use. In one review of 13 patients by Peters et al, ${ }^{62}$ they described the complication in 7 with type 1 diabetes mellitus and two with T2DM. The altered physiology under SGLT2 inhibition can explain this via several mechanisms. With increased glucosuria, there is a relative hypoinsulinemia that leads to lipolysis, along with increased glucagon synthesis, both of which lead to ketogenesis. Additional predisposing factors include food restriction and alcohol intake. The concerning phenomena with euglycemic DKA is the uncoupling of the clinical presentation from symptoms of hyperglycemia. Only $32 \%$ of the patients reviewed by Peters et al presented with vomiting. Thus, these patients are particularly vulnerable as they cannot appropriately monitor the degree of ketosis under the normal mechanisms of symptomatology and glucose monitoring. It may be advisable that patients check their urine for ketones if they begin to feel ill. Euglycemic DKA also presents a dilemma in the postoperative patient as the duration of action of SGLT2 inhibitors is not definitively known. Overall, the rate of ketoacidosis in patients with T2DM is quite low at $\sim 0.5 \%{ }^{63}$

\section{Amputations}

Canagliflozin Cardiovascular Assessment Study (CANVAS) ${ }^{24}$ is an ongoing randomized control study in which more than 4000 patients with T2DM receiving either $100 \mathrm{mg}$ or $300 \mathrm{mg}$ of canagliflozin are being compared with placebo (Table 1). Their current data have detected an increase in lower extremity amputations, predominantly toe amputations. The reported rates in this ongoing trial are three cases per 1000 patientyears in placebo, seven cases per 1000 patient-years in those treated with $100 \mathrm{mg}$ daily of canagliflozin, and five cases per 1000 patient-years in patients receiving $300 \mathrm{mg}$ daily 
of canagliflozin. The European Medicines Agency (EMA) has requested additional analysis to determine whether this is merely a correlation or causal relationship, as the highrisk patients in this study with uncontrolled diabetes and peripheral vascular disease are prone to these complications. The FDA issued a safety announcement regarding the issue, encouraging additional vigilance among providers (http:// www.medscape.com/viewarticle/862009?src=medscapeappipad\&ref=email; accessed on August 29, 2016; MedScape account [free] needed).

\section{Long-term beneficial effects of SGLT2 inhibition \\ Cardiovascular}

Aside from glucose control, and the weight and BP reductions observed with SGLT2 inhibitors such as canagliflozin, the recent EMPA-REG trial demonstrated a benefit of a different SGLT2 inhibitor, empagliflozin, on CV outcomes in patients with T2DM..$^{30}$ EMPA-REG was a multicenter, international, randomized, double-blind, placebo-controlled trial that assessed the effect of empagliflozin versus placebo with regard to a myriad of primary and secondary outcomes. The investigators observed that after a mean follow-up time of $\sim 3$ years, empagliflozin had lower rates of death from CV causes (3.7\% vs $5.9 \%$ in the placebo group, with a $38 \%$ relative risk reduction), hospitalization for heart failure ( $2.7 \%$ vs $4.1 \%$, with a $35 \%$ relative risk reduction), and death from any cause $(5.7 \%$ vs $8.3 \%$, with a $32 \%$ relative risk reduction) ${ }^{49}$

\section{Kidney function}

The report on the EMPA-REG CV findings was followed by an investigation that centered on kidney outcomes in a subset of the EMPA-REG participants. ${ }^{64}$ These patients with nephropathy, defined as an eGFR $>30 \mathrm{~mL} / \mathrm{min} / 1.73 \mathrm{~m}^{2}$, were evaluated for the primary outcome defined as the development of end-stage kidney disease or a doubling of the serum creatinine. In this study, investigators observed that $12.7 \%$ of patients in the empagliflozin group versus $18.8 \%$ in the placebo group (hazard ratio in the empagliflozin group, 0.61 ; $95 \%$ CI, $0.53-0.70 ; P<0.001)$ reached the primary outcome. ${ }^{64}$ Studies of canagliflozin in patients with chronic kidney disease show an early modest decrease in eGFR that stabilizes and returns toward baseline values when canagliflozin is stopped. ${ }^{42}$ Part of this renoprotection is likely linked to the aforementioned changes in tubuloglomerular feedback and minimizing hyperfiltration.

An additional renal protective mechanism of SGLT2 inhibitor may involve inhibition of fibrogenesis. In vitro studies have found that application of D-glucose to either the apical or basolateral side of confluent porcine kidney cells revealed an increase in basolateral fibronectin accumulation. ${ }^{65}$ It would be insightful to investigate how this observation would be changed with application of SGLT2 inhibition. However, a separate study in SGLT2 knockout mice did not show changes to oxidative stress or fibrosis, suggesting that SGLT2 inhibitors modulate mechanisms of fibrosis independent of its receptor protein proper that culminates in protection from oxidative stress. ${ }^{66}$ Other studies have suggested reductions of inflammatory and fibrotic makers under SGLT2 inhibition.

Importantly, the effects of SGLT2 inhibitors require a certain amount of GFR to ensure pharmacologic effect. Given this constraint, benefits with regard to renal prognosis would be cultivated if therapy is initiated early when GFR begins to decline.

Finally, a recent review suggests that these benefits may be a class effect and not just related to empagliflozin, or to the high-risk population enrolled in the EMPA-REG trial. ${ }^{67}$

\section{Completed clinical studies using canagliflozin}

Intervention trials using canagliflozin have centered on several end points, including reduction in urine albumin excretion, reducing proteinuria and reducing end-stage renal disease, and major adverse $\mathrm{CV}$ effects. Table 1 shows specific trials using canagliflozin, enrollment goals, and specific outcomes. To date, a combined analysis of nine studies of canagliflozin conducted prior to FDA approval observed a lower occurrence of major adverse $\mathrm{CV}$ events, including fatal/nonfatal heart attack, fatal/nonfatal stroke, death from CV causes, or hospitalization due to unstable angina, with canagliflozin versus other antihyperglycemic medication ( 18.9 vs 20.5 events per 1000 patient-years) as shown at: http://www.accessdata. fda.gov/drugsatfda docs/nda/2013/204042Orig1s000SumR. pdf (accessed on September 1, 2016). A numeric, but not statistically significant, increase in the occurrence of stroke was observed with canagliflozin versus comparator (6.8 vs 4.6 events per 1000 patient-years) in this preliminary analysis, a curious finding given the BP reduction and something that was observed in EMPA-REG as well, where the comparator group had 10.5 fatal/nonfatal stroke events per 1000 patientyears compared with the empagliflozin arms, which had a rate of 12.3 events per 1000 patient-years. ${ }^{49}$

\section{Clinical caveats with canagliflozin therapy}

The most common side effect ascribed to canagliflozin, and all SGLT2 inhibitors, is mycotic genitourinary tract infections 
because of the glucose in the urine. Fortunately, to date, these appear to be relatively simple to treat and patients can continue the SGLT2 treatment throughout infection and afterward.

A drug capable of lowering BP may do so excessively, and a consequence of SGLT2 inhibition is a symptomatic reduction in BP, particularly upon standing, likely as a result of the diuretic-like effect on circulating blood volume. ${ }^{36}$ Volumerelated effects also include dizziness, nausea, fatigue, and occasionally numerically significant falls in standing SBP of $20 \mathrm{mmHg}$ or more. Hydration is encouraged in patients taking these medications. As mentioned earlier, if treated with a diuretic, clinicians often halve, or hold, diuretic therapy in patients with diabetes when starting canagliflozin, or any of the other SGLT2 inhibiting drugs.

An increase in serum creatinine, not unlike the changes often noted when starting an angiotensin converting enzyme (ACE) inhibitor or an angiotensin receptor blocker, has been observed. These are typically on the order of up to $0.2 \mathrm{mg} / \mathrm{dL}$ increases in serum creatinine over baseline values and are similar to the effects of thiazide diuretics on kidney function in diabetics, tending to return to pretreatment values if the SGLT2 is discontinued. ${ }^{42}$ It may be that SGLT2 inhibitors reduce the hyperfiltration associated with diabetes. ${ }^{68}$

One of the most concerning complications, fortunately uncommon, with canagliflozin usage is normoglycemic ketoacidosis. ${ }^{30}$ This is more likely to occur when managing a type 1 diabetic (for whom, at this time, these drugs are not indicated), in whom insulin dosages are reduced as glucose improves. The relative insulinopenia, along with an increase in glucagon associated with SGLT2 therapy, contributes to the ketoacidosis and it has been reported in some patients with T2DM who are relatively insulin deficient.

\section{Ongoing studies of canagliflozin}

Table 2 lists the ongoing major trials using canagliflozin.

\section{Canagliflozin Cardiovascular Assessment Study}

This is a multinational study comparing the effects of canagliflozin compared with standard of care in type 2 diabetics who have $\mathrm{HbA1C}$ concentrations of $7 \%-10.5 \%$ at enrollment. The trial is active but not recruiting and is assigned the number NCT01032629 at clinical trials website (www. clinicaltrials.gov). There are three arms, with enrolled participants randomized to placebo, canagliflozin $100 \mathrm{mg}$ daily, or canagliflozin $300 \mathrm{mg}$ daily, along with standard care of diabetes. CANVAS included high CV risk participants characterized by either existing symptomatic $\mathrm{CV}$ disease (in the coronary, cerebral, or peripheral circulations) or at high risk of $\mathrm{CV}$ events $(>2.25 \%$ events per year was the estimated risk cutoff level) requiring participants to be in the age group of $\geq 50$ years and to have two of the following: duration of diabetes mellitus (DM) for $>10$ years; $\mathrm{SBP}>140 \mathrm{mmHg}$ or at least one BP medication; current smoker; albuminuria above normal range; or a reduced HDL cholesterol concentration if not symptomatic, as detailed in the design paper. ${ }^{24}$ The primary outcome of CANVAS is the time to occurrence of the first event of death, nonfatal heart attack, and or nonfatal stroke.

\section{Evaluation of the effects of Canagliflozin on Renal and Cardiovascular Outcomes in Participants with Diabetic Nephropathy (CREDENCE)}

This is also a multinational study comparing the effects of $100 \mathrm{mg}$ canagliflozin daily with placebo, with standard care in type 2 diabetics with nephropathy who have HbA1C concentrations of $6.5 \%-12.0 \%$ at enrollment. Nephropathy is defined as having an eGFR of $30-90 \mathrm{~mL} / \mathrm{min} / 1.73 \mathrm{~m}^{2}$ and a urine ACR of $300 \mathrm{mg}$ (albumin)/gram (creatinine). The trial is active and recruiting and is assigned the number NCT02065791 at clinical trials website. The primary outcome of CREDENCE is the time to end-stage kidney disease, doubling of the serum creatinine concentration, or a CV- or renal-related death.

\section{Summary}

SGLT2 inhibitors such as canagliflozin represent an important addition to the management of T2DM. In addition to glucose lowering, they also promote weight loss and reduce BP. Several important caveats attend their use, including the

Table 2 Major ongoing trials using canagliflozin

\begin{tabular}{|c|c|c|c|c|c|c|}
\hline $\begin{array}{l}\text { Age } \\
\text { (years) }\end{array}$ & $\begin{array}{l}\text { Treatment } \\
\text { arms }^{\mathbf{a}}\end{array}$ & Baseline CV risk & Enrolled (n) & Primary outcome & $\begin{array}{l}\text { Anticipated } \\
\text { end point }\end{array}$ & Study name \\
\hline$\geq 30$ & PBO, 100, 300 & $\begin{array}{l}\mathrm{H} / \mathrm{O} C V \text { event or }>50 \text { years old } \\
\text { with high } \mathrm{CV} \text { risk }\end{array}$ & 4331 & MACE & April 2017 & CANVAS $^{24}$ \\
\hline$\geq 30$ & PBO, 100 & Not applicable & Estimated at 4200 & $\begin{array}{l}\text { ESKD; doubling serum } \\
\text { creatinine; renal or CV death }\end{array}$ & January 2020 & CREDENCE $^{69}$ \\
\hline
\end{tabular}

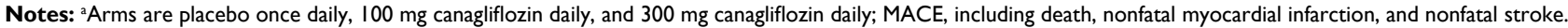
Abbreviations: CANVAS, Canagliflozin Cardiovascular Assessment Study; CREDENCE, Canagliflozin on Renal and Cardiovascular Outcomes in Participants with Diabetic Nephropathy; CV, cardiovascular; ESKD, end-stage kidney disease; H/O, history of; MACE, major adverse CV events; PBO, placebo. 
risk of genital mycotic infections, symptomatic orthostatic BP changes, and a small but clinically important tendency to normoglycemic ketoacidosis. The initial experience from the EMPA-REG studies suggests benefit in heart failure, death, and rates of kidney function loss, which represent important components of the natural history of diabetes. This class of drugs may improve the care of patients with T2DM. The results of several ongoing trials with canagliflozin and other SGLT2 inhibitors should help clarify the place of these drugs in the management of diabetes, particularly in those with comorbidities such as hypertension and CV disease.

\section{Disclosure}

SSS is a board member/advisory panel member for Janssen, Merck, AZ-BMS, BI-Lilly, Salix, Novo, Genesis Biotechnology Group, Intarcia, and Valeant; a stockholder/shareholder for Saturn EMR Decision Support APP and Arkay; a consultant for NIH RO1 DK085212, Struan Grant PI; and has served on speaker's bureaus for Janssen, Merck, Novo, Salix, BI-LILLY, Eisai, AZ-Int'l, and Amgen. JAM and RRT report no conflicts of interest in this work.

\section{References}

1. Centers for Disease Control and Prevention. National Diabetes Statistics Report: Estimates of Diabetes and its Burden in the United States, 2014. 2015. Available from: http://www.cdc.gov/diabetes/pubs/statsreport14/ national-diabetes-report-web.pdf. Accessed August 30, 2016.

2. Ogden CL, Carroll MD, Kit BK, Flegal KM. Prevalence of childhood and adult obesity in the United States, 2011-2012. JAMA. 2014;311(8):806-814.

3. Menke A, Casagrande S, Geiss L, Cowie CC. Prevalence of and trends in diabetes among adults in the United States, 1988-2012. JAMA. 2015;314(10):1021-1029.

4. Centers for Disease Control and Prevention. National Diabetes Statistics Report: Data Sources, Methods, and References for Estimates of Diabetes and Its Burden in the United States, 2014. 2015. Available from: http://www.cdc.gov/diabetes/pdfs/data/2014-report-nationaldiabetes-statistics-report-data-sources.pdf. Accessed August 30, 2016.

5. Committee PP. Standards for medical care in diabetes -2015. Diabetes Care. 2015;38(Suppl 1):S88-S89.

6. Dabelea D, Mayer-Davis EJ, Saydah S, et al. Prevalence of type 1 and type 2 diabetes among children and adolescents from 2001 to 2009. JAMA. 2014;311(17):1778-1786.

7. Imperatore G, Boyle JP, Thompson TJ, et al. Projections of type 1 and type 2 diabetes burden in the U.S. population aged $<20$ years through 2050: dynamic modeling of incidence, mortality, and population growth. Diabetes Care. 2012;35(12):2515-2520.

8. UK Prospective Diabetes Study 6. Complications in newly diagnosed type 2 diabetic patients and their association with different clinical and biochemical risk factors. Diabetes Res. 1990;13(1):1-11.

9. DeFronzo RA. Banting Lecture. From the triumvirate to the ominous octet: a new paradigm for the treatment of type 2 diabetes mellitus. Diabetes. 2009;58(4):773-795.

10. American Diabetes Association. 3. Foundations of care and comprehensive medical evaluation. Diabetes Care. 2016;39(Supp1 1):S23-S35.

11. American Diabetes Association. 7. Approaches to glycemic treatment. Diabetes Care. 2016;39(Suppl 1):S52-S59.

12. Ehrenkranz JR, Lewis NG, Kahn CR, Roth J. Phlorizin: a review. Diabetes Metab Res Rev. 2005;21(1):31-38.
13. Washburn WN. Development of the renal glucose reabsorption inhibitors: a new mechanism for the pharmacotherapy of diabetes mellitus type 2. J Med Chem. 2009;52(7):1785-1794.

14. Hasan FM, Alsahli M, Gerich JE. SGLT2 inhibitors in the treatment of type 2 diabetes. Diabetes Res Clin Pract. 2014;104(3):297-322.

15. Santer R, Calado J. Familial glucosuria and SGLT2: from a mendelian trait to a therapeutic target. Clin JAm Soc Nephrol. 2010;5(1):133-141.

16. Scholl-Burgi S, Santer R, Ehrich JH. Long-term outcome of renal glucosuria type 0 : the original patient and his natural history. Nephrol Dial Transplant. 2004;19(9):2394-2396.

17. Leiter LA, Yoon KH, Arias P, et al. Canagliflozin provides durable glycemic improvements and body weight reduction over 104 weeks versus glimepiride in patients with type 2 diabetes on metformin: a randomized, double-blind, phase 3 study. Diabetes Care. 2015;38(3):355-364.

18. Wright EM. Renal Na(+)-glucose cotransporters. Am J Physiol Renal Physiol. 2001;280(1):F10-F18.

19. Kanai Y, Lee WS, You G, Brown D, Hediger MA. The human kidney low affinity $\mathrm{Na}+$ /glucose cotransporter SGLT2. J Clin Invest. 1994; 93:397-404.

20. Stenlof K, Cefalu WT, Kim KA, et al. Efficacy and safety of canagliflozin monotherapy in subjects with type 2 diabetes mellitus inadequately controlled with diet and exercise. Diabetes Obes Metab. 2013;15(4):372-382.

21. Inagaki N, Kondo K, Yoshinari T, Takahashi N, Susuta Y, Kuki H. Efficacy and safety of canagliflozin monotherapy in Japanese patients with type 2 diabetes inadequately controlled with diet and exercise: a 24-week, randomized, double-blind, placebo-controlled, Phase III study. Expert Opin Pharmacother. 2014;15(11):1501-1515.

22. Forst T, Guthrie R, Goldenberg R, et al. Efficacy and safety of canagliflozin over 52 weeks in patients with type 2 diabetes on background metformin and pioglitazone. Diabetes Obes Metab. 2014;16(5):467-477.

23. Lavalle-Gonzalez FJ, Januszewicz A, Davidson J, et al. Efficacy and safety of canagliflozin compared with placebo and sitagliptin in patients with type 2 diabetes on background metformin monotherapy: a randomised trial. Diabetologia. 2013;56(12):2582-2592.

24. Neal B, Perkovic V, de Zeeuw D, et al. Rationale, design, and baseline characteristics of the Canagliflozin Cardiovascular Assessment Study (CANVAS) - a randomized placebo-controlled trial. Am Heart J. 2013;166(2): 217-223.e11.

25. Wilding JP, Charpentier G, Hollander P, et al. Efficacy and safety of canagliflozin in patients with type 2 diabetes mellitus inadequately controlled with metformin and sulphonylurea: a randomised trial. Int J Clin Pract. 2013;67(12):1267-1282.

26. Cefalu WT, Leiter LA, Yoon KH, et al. Efficacy and safety of canagliflozin versus glimepiride in patients with type 2 diabetes inadequately controlled with metformin (CANTATA-SU): 52 week results from a randomised, double-blind, phase 3 non-inferiority trial. Lancet. 2013;382(9896):941-950.

27. Langslet GC, Cefalu WT, Yoon KH, et al. Canagliflozin demonstrates durable glycaemic improvements over 104 weeks compared with glimepiride in subjects with type 2 diabetes mellitus on metformin. European Association for the Study of Diabetes (EASD); September 23-27, 2013; Barcelona, Spain.

28. Schernthaner G, Gross JL, Rosenstock J, et al. Canagliflozin compared with sitagliptin for patients with type 2 diabetes who do not have adequate glycemic control with metformin plus sulfonylurea: a 52-week randomized trial. Diabetes Care. 2013;36(9):2508-2515.

29. Chen M, Xie CG, Gao H, Zheng H, Chen Q, Fang JQ. Comparative effectiveness of sodium-glucose co-transporter 2 inhibitors for controlling hyperglycaemia in patients with type 2 diabetes: protocol for a systematic review and network meta-analysis. BMJ Open. 2016;6(1):e010252.

30. Inzucchi SE, Zinman B, Wanner C, et al. SGLT-2 inhibitors and cardiovascular risk: proposed pathways and review of ongoing outcome trials. Diab Vasc Dis Res. 2015;12(2):90-100.

31. Cefalu WT, Stenlof K, Leiter LA, et al. Effects of canagliflozin on body weight and relationship to HbA1c and blood pressure changes in patients with type 2 diabetes. Diabetologia. 2015;58(6):1183-1187.

32. Maliha G, Townsend RR. SGLT2 inhibitors: their potential reduction in blood pressure. J Am Soc Hypertens. 2015;9(1):48-53. 
33. Baker WL, Smyth LR, Riche DM, Bourret EM, Chamberlin KW, White WB. Effects of sodium-glucose co-transporter 2 inhibitors on blood pressure: a systematic review and meta-analysis. J Am Soc Hypertens. 2014;8(4):262-275.

34. Weir MR, Januszewicz A, Gilbert RE, et al. Effect of canagliflozin on blood pressure and adverse events related to osmotic diuresis and reduced intravascular volume in patients with type 2 diabetes mellitus. J Clin Hypertens (Greenwich). 2014;16(12):875-882.

35. Townsend RR, Machin I, Ren J, et al. Reductions in mean 24-hour ambulatory blood pressure after 6-week treatment with canagliflozin in patients with type 2 diabetes mellitus and hypertension. J Clin Hypertens (Greenwich). 2016;18(1):43-52.

36. Lambers Heerspink HJ, de ZD, Wie L, Leslie B, List J. Dapagliflozin a glucose-regulating drug with diuretic properties in subjects with type 2 diabetes. Diabetes Obes Metab. 2013;15(9):853-862.

37. Osorio H, Coronel I, Arellano A, et al. Sodium-glucose cotransporter inhibition prevents oxidative stress in the kidney of diabetic rats. Oxid Med Cell Longev. 2012;2012:542042.

38. Malatiali S, Francis I, Barac-Nieto M. Phlorizin prevents glomerular hyperfiltration but not hypertrophy in diabetic rats. Exp Diabetes Res. 2008;2008:305403.

39. Kalra S, Singh V, Nagrale D. Sodium-glucose cotransporter-2 inhibition and the glomerulus: a review. Adv Ther. 2016;33(9):1502-1518.

40. Zanoli L, Granata A, Lentini P, et al. Sodium-glucose linked transporter-2 inhibitors in chronic kidney disease. ScientificWorldJournal. 2015;2015:317507.

41. Yale JF, Bakris G, Cariou B, et al. Efficacy and safety of canagliflozin in subjects with type 2 diabetes and chronic kidney disease. Diabetes Obes Metab. 2013;15(5):463-473.

42. Perkovic V, Jardine M, Vijapurkar U, Meininger G. Renal effects of canagliflozin in type 2 diabetes mellitus. Curr Med Res Opin. 2015; 31(12):2219-2231.

43. Davies MJ, Trujillo A, Vijapurkar U, Damaraju CV, Meininger G. Effect of canagliflozin on serum uric acid in patients with type 2 diabetes mellitus. Diabetes Obes Metab. 2015;17(4):426-429.

44. Caulfield MJ, Munroe PB, O’Neill D, et al. SLC2A9 is a high-capacity urate transporter in humans. PLoS Med. 2008;5(10):e197.

45. Bjornstad P, Lanaspa MA, Ishimoto T, et al. Fructose and uric acid in diabetic nephropathy. Diabetologia. 2015;58(9):1993-2002.

46. Vasilakou D, Karagiannis T, Athanasiadou E, et al. Sodium-glucose cotransporter 2 inhibitors for type 2 diabetes: a systematic review and meta-analysis. Ann Intern Med. 2013;159(4):262-274.

47. Karagiannis T, Liakos A, Bekiari E, et al. Efficacy and safety of onceweekly glucagon-like peptide 1 receptor agonists for the management of type 2 diabetes: a systematic review and meta-analysis of randomized controlled trials. Diabetes Obes Metab. 2015;17(11):1065-1074.

48. Patrick AW, Hepburn DA, Swainson CP, Frier BM. Changes in renal function during acute insulin-induced hypoglycaemia in patients with type 1 diabetes. Diabet Med. 1992;9(2):150-155.

49. Zinman B, Wanner C, Lachin JM, et al. Empagliflozin, cardiovascular outcomes, and mortality in type 2 diabetes. $N$ Engl J Med. 2015; 373(22):2117-2128.

50. Merovci A, Solis-Herrera C, Daniele G, et al. Dapagliflozin improves muscle insulin sensitivity but enhances endogenous glucose production. J Clin Invest. 2014;124(2):509-514.

51. Rosiak M, Grzeszczak S, Kosior DA, Postula M. Emerging treatments in type 2 diabetes: focus on canagliflozin. Ther Clin Risk Manag. 2014; 10:683-689.

52. Sha S, Polidori D, Heise T, et al. Effect of the sodium glucose cotransporter 2 inhibitor canagliflozin on plasma volume in patients with type 2 diabetes mellitus. Diabetes Obes Metab. 2014;16(11):1087-1095.

53. Foote C, Perkovic V, Neal B. Effects of SGLT2 inhibitors on cardiovascular outcomes. Diab Vasc Dis Res. 2012;9(2):117-123.
54. Nishimura R, Tanaka Y, Koiwai K, et al. Effect of empagliflozin monotherapy on postprandial glucose and 24-hour glucose variability in Japanese patients with type 2 diabetes mellitus: a randomized, double-blind, placebo-controlled, 4-week study. Cardiovasc Diabetol. 2015;14:11.

55. Jojima T, Tomotsune T, Iijima T, Akimoto K, Suzuki K, Aso Y. Empagliflozin (an SGLT2 inhibitor), alone or in combination with linagliptin (a DPP-4 inhibitor), prevents steatohepatitis in a novel mouse model of non-alcoholic steatohepatitis and diabetes. Diabetol Metab Syndr. 2016;8:45.

56. Bolinder J, Ljunggren O, Kullberg J, et al. Effects of dapagliflozin on body weight, total fat mass, and regional adipose tissue distribution in patients with type 2 diabetes mellitus with inadequate glycemic control on metformin. J Clin Endocrinol Metab. 2012;97(3):1020-1031.

57. Nyirjesy P, Zhao Y, Ways K, Usiskin K. Evaluation of vulvovaginal symptoms and Candida colonization in women with type 2 diabetes mellitus treated with canagliflozin, a sodium glucose co-transporter 2 inhibitor. Curr Med Res Opin. 2012;28(7):1173-1178.

58. Kohan DE, Fioretto P, Tang W, List JF. Long-term study of patients with type 2 diabetes and moderate renal impairment shows that dapagliflozin reduces weight and blood pressure but does not improve glycemic control. Kidney Int. 2014;85(4):962-971.

59. Meier C, Schwartz AV, Egger A, Lecka-Czernik B. Effects of diabetes drugs on the skeleton. Bone. 2016;82:93-100.

60. Taylor SI, Blau JE, Rother KI. Possible adverse effects of SGLT2 inhibitors on bone. Lancet Diabetes Endocrinol. 2015;3(1):8-10.

61. Bilezikian JP, Watts NB, Usiskin K, et al. Evaluation of bone mineral density and bone biomarkers in patients with type 2 diabetes treated with canagliflozin. J Clin Endocrinol Metab. 2016;101(1):44-51.

62. Peters AL, Buschur EO, Buse JB, Cohan P, Diner JC, Hirsch IB Euglycemic diabetic ketoacidosis: a potential complication of treatment with sodium-glucose cotransporter 2 inhibition. Diabetes Care. 2015;38(9):1687-1693.

63. Handelsman Y, Henry RR, Bloomgarden ZT, et al. American Association of Clinical Endocrinologists and American College of Endocrinology position statement on the association of Sglt-2 inhibitors and diabetic ketoacidosis. Endocr Pract. 2016;22(6):753-762.

64. Wanner C, Inzucchi SE, Lachin JM, et al. Empagliflozin and progression of kidney disease in type 2 diabetes. N Engl J Med. 2016;375(4): 323-334.

65. Morrisey K, Steadman R, Williams JD, Phillips AO. Renal proximal tubular cell fibronectin accumulation in response to glucose is polyol pathway dependent. Kidney Int. 1999;55(1):160-167.

66. Vallon V, Rose M, Gerasimova M, et al. Knockout of Na-glucose transporter SGLT2 attenuates hyperglycemia and glomerular hyperfiltration but not kidney growth or injury in diabetes mellitus. Am J Physiol Renal Physiol. 2013;304(2):F156-F167.

67. Wu JH, Foote C, Blomster J, et al. Effects of sodium-glucose cotransporter-2 inhibitors on cardiovascular events, death, and major safety outcomes in adults with type 2 diabetes: a systematic review and metaanalysis. Lancet Diabetes Endocrinol. 2016;4(5):411-419.

68. De Nicola L, Gabbai FB, Liberti ME, Sagliocca A, Conte G, Minutolo R. Sodium/glucose cotransporter 2 inhibitors and prevention of diabetic nephropathy: targeting the renal tubule in diabetes. Am J Kidney Dis. 2014;64(1):16-24.

69. Janssen Research and Development, LLC. Evaluation of the Effects of Canagliflozin on Renal and Cardiovascular Outcomes in Participants With Diabetic Nephropathy (CREDENCE). Available from: https://clinicaltrials.gov/ct2/show/NCT02065791. NLM identifier: NCT02065791. Accessed November 29, 2016.

70. Schwartz SS, Ahmed I. Sodium-glucose cotransporter 2 inhibitors: an evidence-based practice approach to their use in the natural history of type 2 diabetes. Curr Med Res Opin. 2016;32(5):907-919. 


\section{Publish your work in this journal}

Vascular Health and Risk Management is an international, peerreviewed journal of therapeutics and risk management, focusing on concise rapid reporting of clinical studies on the processes involved in the maintenance of vascular health; the monitoring, prevention and treatment of vascular disease and its sequelae; and the involvement of metabolic disorders, particularly diabetes. This journal is indexed on PubMed Central and MedLine. The manuscript management system is completely online and includes a very quick and fair peer-review system, which is all easy to use. Visit http://www.dovepress.com/ testimonials.php to read real quotes from published authors. 